Revue d'histoire de l'Amérique française

REVUE D.HISTOIRE DE L'AMÉRIQUE FRANÇAISE

\title{
Les insuffisances d'une analyse purement historique des droits des peuples autochtones
}

\section{Michel Morin}

Volume 57, numéro 2, automne 2003

URI : https://id.erudit.org/iderudit/009144ar

DOI : https://doi.org/10.7202/009144ar

Aller au sommaire du numéro

Éditeur(s)

Institut d'histoire de l'Amérique française

ISSN

0035-2357 (imprimé)

1492-1383 (numérique)

Découvrir la revue

Citer cette note

Morin, M. (2003). Les insuffisances d'une analyse purement historique des droits des peuples autochtones. Revue d'histoire de l'Amérique française, 57(2), 237-254. https://doi.org/10.7202/009144ar d'utilisation que vous pouvez consulter en ligne. 


\section{Note de recherche}

\section{Les insuffisances d'une analyse purement historique des droits des peuples autochtones}

MICHEL MORIN ${ }^{1}$

Faculté de droit

Université de Montréal

\section{INTRODUCTION}

Les publications récentes portant sur l'histoire des peuples autochtones sont d'une richesse extraordinaire. Elles remettent en question bien des idées reçues, en exhumant des archives des informations nouvelles et en faisant appel à des approches novatrices. Dans certains cas, elles contiennent toutefois des affirmations inexactes voire caricaturales portant sur des questions juridiques ${ }^{2}$. Cette mauvaise perception risque de fausser les conclusions portant sur l'existence de droits ancestraux ou issus de traité ${ }^{3}$. En effet, à défaut de faire des vérifications minimales, on risque de méconnaître les notions juridiques en se fondant exclusivement sur des conceptions historiques.

Bien entendu, de nombreux écrits juridiques témoignent eux aussi d'une méconnaissance de la littérature historique, mais cet aspect du

1. L'auteur tient à remercier ses collègues Jean-Paul Lacasse et Jean Leclair de leurs précieux commentaires, ainsi que Denis Blanchette, étudiant à la maîtrise à la Faculté de droit de l’Université de Montréal, pour son aide dans la préparation de la version définitive.

2. Donald Fyson avait déjà signalé ce problème: "Les historiens du Québec face au droit ", Revue juridique Thémis, 34 (2000): 295-328, 306-307.

3. L'article 35 (1) de la Loi constitutionnelle de 1982 (annexe B de la Loi de 1982 sur le Canada, 1982, c. 11 (R.-U.), se lit comme suit: «Les droits existants - ancestraux ou issus de traités- des peuples autochtones du Canada sont reconnus et confirmés». 
problème ne retiendra pas notre attention ${ }^{4}$. Nous insisterons plutôt sur le traitement insatisfaisant ou discutable de certaines questions juridiques, en prenant pour exemple des études portant sur les fondements historiques des droits des Autochtones du Québec et des Provinces maritimes à l'époque de la Conquête ou du Régime français ${ }^{5}$. Toutefois, notre objectif n'est pas de faire une revue de la littérature pertinente ou d'analyser l'ensemble de la jurisprudence. Nous présenterons plutôt les principes dont il aurait été important de tenir compte, en citant le cas échéant la décision rendue par la cour la plus élevée dans la hiérarchie judiciaire, car c'est elle qui fait autorité, ou encore l'approche retenue par des juristes.

Nous ne chercherons pas non plus à déterminer si le statut d'un auteur (universitaire ou consultant indépendant, spécialiste ou non des questions autochtones, anthropologue ou historien) ou le fait qu'une étude ait été commanditée par le gouvernement ou par une communauté autochtone accentue le problème discuté ci-dessous. Les risques d'abus sont évidents, d'un côté comme de l'autre d'ailleurs ${ }^{6}$; mais, à l'instar d'Arthur Ray, il faut résister à la tentation de considérer tous les

4. En Colombie-Britannique, le juge en chef de la Cour suprême de la ColombieBritannique, Allan McEachern a rendu jugement en première instance dans l'affaire Delgamuukw c. Bristish-Columbia, [1991] 3 W.W.R. 97. Des auteurs ont dénoncé sa conception réductrice de l'histoire consistant à "copier-coller» des extraits de documents sans aucune mise en contexte ou interprétation: Robin Fisher, "Judging History: Reflections on the Reasons for Judgment in Delgamuukw v. B.C.», B. C. Studies, 95 (1992): 43-54; Joel R. Fortune, "Comments: Construing Delgamuukw: Legal Arguments, Historical Argumentation, and the Philosophy of History », University of Toronto Faculty of Law Review, 51 (1993): 80. Par la suite, la Cour suprême du Canada a ordonné un nouveau procès ([1997] 3 R.C.S. 1010) en raison des préjugés du juge en chef McEachern à l'égard des récits oraux. Plus généralement, un déluge de publications ont critiqué l'ethnocentrisme caractérisant son jugement; elles sont résumées dans Brian Thom, "Aboriginal Rights and Title in Canada After Delagamuukw: Part One, Oral Traditions and Anthropological Evidence in the Courtroom", Native Studies Review, 14,1 (2001): 1-26 et «Aboriginal Rights and Title in Canada After Delagamuukw: Part Two, Anthropological Perspectives on Rights, Tests, Infringement \& Justification ", Native Studies Review, 14 :2 (2001) : 1-42.

5. Nous ne traiterons pas de l'abondante littérature suscitée par l'affaire R. c. Marshall, [1999] 3 R.C.S. 456 et 533 ; voir notamment les publications citées dans Michel Morin, "Quelques réflexions sur le rôle de l'histoire dans la détermination des droits ancestraux ou issus de traités", Revue juridique Thémis, 34 (2000): 329-368, 358-364; Arthur J. Ray, «Regina v Marshall: Native History, the Judiciary and the Public", Acadiensis, $29: 2$ (2000): 138-146; Ken Coates, The Marshall Decision and Native Rights (Montréal, McGill-Queen's University Press, 2000); William C. Wicken, Mi'kmaq Treaties on Trial, History, Land and Donald Marshall Junior (Toronto, University of Toronto Press, 2002) notamment $\mathrm{x}$-xi, 10-14, 20-21, 67-70, 84-85, 140-143 et 225-236. Cette excellente analyse, rédigée par un historien, explique clairement le rôle de l'expert déposant devant un tribunal et les possibilités d'interprétations divergentes de la documentation historique.

6. Alain Beaulieu, «Les pièges de la judiciarisation de l'histoire autochtone», Revue d'histoire de l'Amérique française, 53,4 (hiver 2000): 541-551. 
experts qui déposent en cour comme des «hommes de main, des chacals ou des putains ${ }^{7} »$. Les exemples ne manquent d'ailleurs pas d'études rigoureuses qui reconnaissent les limites de leur démarche ${ }^{8}$.

\section{LA MINIMISATION DES ÉLÉMENTS JURIDIQUES}

En ne prêtant pas attention à la terminologie juridique, les chercheurs risquent de négliger une dimension importante du passé. C’est ce que montre l'étude de la controverse ayant entouré la reconnaissance par les tribunaux du traité dit de Murray. De même, il est difficile de comprendre pourquoi certaines publications font abstraction des critères souples posés par la jurisprudence contemporaine en ce qui concerne la continuité entre la situation actuelle d'un peuple autochtone et celle dans laquelle se trouvaient placés ses ancêtres.

\section{La terminologie juridique et le traité de Murray}

Les risques d'erreurs que comporte l'utilisation de concepts juridiques anciens peuvent être illustrés par une étude récente de Denys Delâge et d'Étienne Gilbert portant sur les procès de 1760 à 1820 où au moins l'une des parties est autochtone. Les auteurs semblent ignorer qu'en 1774, la France et le Québec n’ont pas encore adopté de code civil ${ }^{9}$. Ils supposent en outre qu’à cette époque, le «bénéfice du clergé » fait suite à la demande d'un prêtre de gracier un condamné à mort. En réalité, dans certains cas, cette règle de droit anglais permet au juge d'accorder la vie sauve à l'accusé non récidiviste; il y a bien longtemps que le clergé ne joue plus aucun rôle à cette étape de la procédure ${ }^{10}$. Ces auteurs croient également qu'il est "illégal» de vivre en union de fait, alors qu'aucune sanction pénale n'est imposée dans ce cas, même si cette relation ne confère aucun droit aux partenaires ${ }^{11}$.

7. Arthur J. Ray, «Native History on Trial: Confessions of an Expert Witness", Canadian Historical Review, 84 (2003): 253-273, 269 (notre traduction).

8. Même si nous ne partageons pas toujours leurs conclusions, il nous semble que les divers rapports d'experts qui ont été reproduits dans Denis Vaugeois, dir., Les Hurons de Lorette (Sillery, Septentrion, 1996), constituent de bons exemples d'études historiques rigoureuses.

9. Denys Delâge et Étienne Gilbert, «La justice coloniale britannique et les Amérindiens au Québec 1760-1820, I: En terres amérindiennes", Recherches amérindiennes au Québec, 32,1 (2002): 63-82, 66 .

10. Denys Delâge et Étienne Gilbert, «La justice coloniale britannique et les Amérindiens au Québec 1760-1820, II : En territoire colonial», Recherches amérindiennes au Québec, 32,2 (2002): 107-117, 108; pour plus de détails, voir J. H. Baker, Introduction to English Legal History (Londres, Butterworths, 1990), 586-589.

11. D. Delâge et E. Gilbert, ibid., 111. À notre connaissance, les règles du droit anglais imposant une peine pour adultère ou pour avoir enfanté hors mariage n'ont jamais été 
Mais au-delà de ces points de détail, il existe des termes polysémiques dont la portée est éminemment discutable. Pour clarifier celle-ci, on ne peut faire l'économie de l'analyse des documents juridiques. Ainsi, en Nouvelle-France et en Nouvelle-Angleterre, au xvII siècle, divers actes officiels applicables aux représentants de la couronne emploient les termes "princes», "nations» ou "alliés " pour désigner les Autochtones. $\mathrm{Au}$ minimum, ils reconnaissent à cette occasion leur statut collectif, voire l'indépendance dont ils jouissent. De même, l'emploi du terme "traité» ou d'expressions telles que "articles de la paix» ou "faire la paix" correspond à une relation de nature internationale, quand bien même celle-ci présenterait des différences marquées par rapport à celles qu'entretiennent les puissances européennes ${ }^{12}$. Dès lors, peut-on donner son "opinion d'historien" sur la conclusion d'un traité sans s'interroger sur la signification juridique de ce concept dans le passé, comme le font Denys Delâge et Jean-Pierre Sawaya ${ }^{13}$ ?

Certes, en analysant méthodiquement le processus de négociation et les documents d'archives, il est possible de dégager les caractéristiques habituelles de ces ententes. Telle est l'approche employée par Marcel Trudel, qui distingue quatre étapes dans la conclusion d'un traité entre peuples autochtones : l'invitation, la rencontre à l'orée du bois, le conseil (où se déroulent les négociations) et la ratification ultérieure ${ }^{14}$. Or, il n'est pas toujours facile de reconstituer avec précision les différentes étapes de ce processus.

Ce problème est à l'origine de la controverse qui a entouré le traité dit de Murray, ou plus exactement le certificat délivré par celui-ci en 1760 aux Hurons de Lorette. Le brigadier-général britannique y certifie que le chef des Hurons est venu au nom de sa nation "pour se soumettre à sa

appliquées au Québec: voir William Blackstone, Commentaries on the Laws of England in Four Books, New Edition (London, Apollo Press, vol. IV, 1813, c. IV), 58-59.

12. Voir généralement Michel Morin, L'Usurpation de la souveraineté autochtone (Montréal, Boréal, 1997), chap. 2 à 5 ; Gilles Havard, Empire et métissages, Indiens et Français dans le Pays d'en haut, 1660-1715 (Sillery/Paris, Septentrion/Presses de l'Université Paris-Sorbonne, 2003), 482490; Alain Beaulieu, "La paix de 1624, Les enjeux géo-politiques du premier traité francoiroquois", dans Alain Beaulieu, dir., Guerre et Paix en Nouvelle-France (Sainte-Foy, Les Éditions du GID, 2003), 53-101; Maxime Gohier, "Onontio le Médiateur. La diplomatie franco-amérindienne au lendemain de la Grande Paix de Montréal », dans ibid., 103-139 (notamment p. 129, note 5).

13. Denys Delâge et Jean-Pierre Sawaya, Les traités des Sept-Feux avec les Britanniques. Droits et pièges d'un héritage colonial au Québec (Sillery, Septentrion, 2001), 57.

14. Voir Marcel Trudel, «Les Hurons et Murray en 1760: Un "traité” qui n'est qu’un laissezpasser», dans D. Vaugeois, op. cit., 132-158. 
Majesté britannique, et faire la paix»; en outre, il a été reçu sous la "protection» de Murray, "avec toute sa tribu». Les militaires anglais doivent donc s'abstenir de molester les Hurons, qui sont «reçus aux mêmes conditions que les Canadiens, étant permis le libre exercice de leur religion, leur coutume, et la liberté de commerce avec les garnisons anglaises ${ }^{15}$ ». Ce document fait donc état d'une relation de nation à nation, même si l'une d'elles se soumet à l'autre et est reçue aux mêmes conditions que les Canadiens. Puis, il reconnaît une liberté de circulation, de religion et de commerce avec les garnisons anglaises. Il ne semble pas qu'il ait donné lieu à des formalités particulières, contrairement à ce qui se produit habituellement lors des négociations avec les Nations autochtones. En effet, cette rencontre imprévue semble avoir été fort brève, afin de ne pas retarder les opérations militaires qui se déroulent ce jour-là.

L'expression «leurs coutumes» a suscité des interprétations étonnantes. Nelson-Martin Dawson et Éric Tremblay y ont vu une référence à la Coutume de Paris, qui n’a pourtant jamais été appliquée dans le village des Hurons ${ }^{16}$. Ils ont, pour une fois, donné une portée juridique à un terme qui était employé dans un sens plus large. Pour leur part, Delâge et Sawaya estiment que cette garantie est similaire à celle de la Capitulation de Montréal, par laquelle les Britanniques se seraient engagés à respecter les coutumes du peuple conquis ${ }^{17}$. Pourtant, l'article 42 constitue simplement une proposition des Français au commandant Jeffery Amherst. Celui-ci leur répond que les Canadiens deviennent sujets du roi; le général britannique s'abstient ainsi de prendre quelque engagement que ce soit à l'égard de leur système juridique ${ }^{18}$.

Le certificat de Murray a été soumis à l'attention des tribunaux dans l'affaire R.c. Sioui ${ }^{19}$. En effet, les frères Sioui ont été accusés d'avoir violé la réglementation provinciale en coupant du bois, en campant et en faisant un feu dans le parc provincial de la Jacques Cartier. Ils allèguent avoir alors pratiqué des rites ancestraux et religieux hurons qui sont

15. Nous citons la traduction littérale de Nelson-Martin Dawson et Éric Tremblay, «La preuve historique dans le cadre des procès relatifs au droit autochtone et aux crimes contre l'humanité", Revue de droit de l'Université de Sherbrooke, 30 (2000): 377-406, 394.

16. Ibid., 398; pour une critique plus élaborée de cette position, voir M. Morin, "Quelques réflexions...", op. cit., 352, à la note 69 .

17. D. Delâge et J.-P. Sawaya, op. cit., 58.

18. Adam Shortt et Arthur Doughty, dir., Documents relatifs à l'histoire constitutionnelle $d u$ Canada, 1759-1791, 2e éd. (Ottawa, Imprimeur de la Reine, 1921), 5.

19. [1990] 1 R.C.S. 1025. 
protégés par le certificat rédigé en 1760 à titre de coutume. En 1990, la Cour suprême du Canada leur donne raison. Elle conclut que ce document constitue la «matérialisation» d'une entente orale ${ }^{20}$ qui a été ratifiée par la suite à Caughnawage (devenu Kahnawake), lors d'une conférence tenue les 15 et 16 septembre, quelques jours après la Capitulation des forces françaises. Celle-ci réunissait des représentants des nations autochtones domiciliées en Nouvelle-France, des Britanniques et des Iroquois. Selon toute vraisemblance, les Hurons ont d'ailleurs été informés des négociations qui s'étaient déroulées le mois précédent à Oswegatchie entre William Johnson et les Iroquois domiciliés ${ }^{21}$. Dans ces conditions, pour reprendre les termes du juge Lamer, «il est raisonnable de voir dans la présentation des ceintures lors de la conférence du 16 septembre une ratification solennelle de l'accord de paix conclu quelques jours plus tôt » avec Murray ${ }^{22}$. Mais quelques lignes plus loin, il affirme que «le document du 5 septembre 1760 est un traité au sens de l'art. 88 de la Loi sur les Indiens ${ }^{23}$. Ce faisant, il crée une confusion entre l'entente et le certificat remis aux Hurons.

Tous les historiens s'entendent pour dire qu'un traité a bel et bien été conclu à Kahnawake avec huit nations domiciliées au Canada, dont les Hurons, même si ceux-ci ne semblent pas avoir été présents ${ }^{24}$. Mais plusieurs estiment que le certificat délivré par Murray ou les discussions qui ont précédé cet événement ne sauraient être considérés comme un accord de cette nature ${ }^{25}$. En ce qui concerne l'absence de négociations, la rencontre avec Murray a fait l'objet de deux comptes rendus sommaires et parfois contradictoires qui laissent place à plusieurs interprétations. La tradition orale huronne ne semble d'ailleurs pas avoir gardé le souvenir d'un traité, même si le certificat a été confié à un notaire en 1810. Par la suite, une version imprimée a été déposée devant l'Assemblée législative du Bas-Canada pour appuyer une revendication territoriale, apparem-

20. Ibid., 1057.

21. Voir Alain Beaulieu, "Les Hurons et la Conquête, Un nouvel éclairage sur le "traité Murray”", Recherches amérindiennes au Québec, 30,3 (2000): 53-63, 59-60.

22. R. c. Sioui, op. cit., 1060.

23. Ibid.

24. Alain Beaulieu, "Les garanties d'un traité disparu: le traité d'Oswegatchie, 30 août 1760 », R.J.T., 34 (2000) : 369-408, 387-389; M. Trudel, op. cit., 158; Denis Vaugeois, La fin des alliances franco-indiennes, Enquête sur un sauf-conduit de 1760 devenu un traité en 1990 (Montréal/ Québec, Boréal/Septentrion, 1995), 167. Le nombre de huit nations est employé dans le compte rendu de la conférence (ibid., 214-221).

25. A. Beaulieu, "Les Hurons...», op. cit. ; N.-M. Dawson et É. Tremblay, "La preuve historique... », op. cit. ; D. Vaugeois, La fin..., op. cit. 
ment sans plus de commentaires ${ }^{26}$. Quoi qu'il en soit, toutes ces versions retiennent que les Hurons ont fait la paix; dans le contexte, il ne peut s'agir que d'une paix avec les Britanniques ${ }^{27}$. Celle-ci constitue le contenu minimal de l'entente, laquelle n'a pas d'équivalent dans le cas des sujets du roi de France qui déposent les armes. Nous croyons qu'il convient de parler d'un engagement préliminaire de paix, qui a été ratifié à Kahnawake quelques jours plus tard. S'il ne constitue pas à lui seul un traité, il s'inscrit dans le processus qui mène à la conclusion de celui-ci ${ }^{28}$.

Le pouvoir de Murray de conclure des traités a également été mis en doute, car cette question relevait du surintendant des affaires indiennes. Pour cette raison, plusieurs historiens ne croient pas qu'il ait eu l'intention de conclure une telle entente ${ }^{29}$. Il est bien possible qu'il ne se soit pas interrogé très longuement sur la portée exacte des termes qu'il employait. En bon militaire, il lui suffisait de s'assurer que les Hurons cessaient de se battre et ne seraient pas inquiétés durant leur retour. Les autres questions pouvaient très bien être réglées plus tard par William Johnson, ce qui sera fait les 15 et 16 septembre à Kahnawake.

Une telle façon de procéder est attestée en Nouvelle-Écosse et au Cap Breton. Dans un procès où des Micmacs revendiquent des droits ancestraux sur l'île de Terre-Neuve, le professeur Stephen Patterson a agi comme expert pour le compte du gouvernement. Un extrait de son rapport est reproduit dans le jugement, qui, incidemment, rejette la revendication des Micmacs. M. Patterson y explique que les officiers militaires britanniques pouvaient accepter la reddition de forces ennemies, autochtones ou françaises, en agréant au besoin à leurs demandes. De telles ententes sont qualifiées de «traités militaires». Elles constituent pour cet auteur "une soumission préliminaire des peuples autochtones à la couronne britannique», qui doit être confirmée et précisée ultérieurement par une autorité supérieure (en l'occurrence, le gouverneur et commandant en chef $)^{30}$. Telle nous semble très exactement la situation

26. David Schulze, "The Murray Treaty of 1760: The Original Document Discovered», Canadian Native Law Reporter, 1 [1998]: 1-13. Il a fallu attendre jusqu'en 1996 pour que l'original soit découvert par cet auteur dans les archives du notaire Barthélémy Faribault.

27. Voir toutefois N.-M. Dawson et É. Tremblay, «La preuve historique...., op. cit., 394-397.

28. On trouvera une présentation plus complète de ce débat dans M. Morin, "Quelques réflexions...», op. cit., 349-355.

29. A. Beaulieu, supra, note 21 ; N.-M. Dawson et É. Tremblay, "La preuve historique... », op. cit.; D. Vaugeois, La fin..., op. cit.

30. Newfoundland (Minister of Government Services and Lands) c. Drew, [2003] N.J. No. 177 (C.S.L.T.-N.) (QL Systems), par. 752 et 1047. 
des Hurons. La paix préliminaire attestée par le certificat permet de les rattacher au traité conclu à Kahnawake quelques jours plus tard et de préciser les droits qui leur ont été reconnus au moment de la Conquête.

Dans l'ensemble, si la nature exacte du certificat délivré par Murray et le rôle joué par celui-ci sont discutables, il n'est guère contestable que les nations domiciliées et les Britanniques ont conclu un traité de paix en 1760. D’un point de vue historique, il aurait été préférable que la Cour suprême du Canada parle du traité de Kahnawake. Mais le gouvernement et les accusés ont fait porter le débat exclusivement sur le certificat. L'Assemblée des Premières Nations a toutefois été autorisée à présenter des arguments distincts devant la Cour suprême du Canada à titre d'intervenante, car elle n'était pas visée par la poursuite initiale. C'est elle qui a attiré l'attention de la Cour sur la conférence de Kahnawake $^{31}$. Dans ces conditions, il aurait été imprudent de se prononcer sur la portée exacte de celle-ci. À moins d'ordonner la tenue d'un nouveau procès dont l'issue était de toute manière prévisible, la Cour n'avait d'autre choix que de rattacher ses conclusions au document invoqué par les Hurons. Même s'il s'agit indubitablement d'un raccourci historique, cette solution était la plus appropriée dans les circonstances.

\section{Le problème de la discontinuité}

Dans l'arrêt Sioui, le juge Lamer examine le cas d'un groupe de Hurons venu s'établir aux environs de Québec en 1650. Il déclare que «sa présence relativement récente dans la région de Lorette suggère " qu'ils «n'avaient pas la possession historique de ces terres ${ }^{32}$ ». Telle est peut-être l'origine d'une croyance répandue selon laquelle un lien de continuité direct doit être établi entre les Autochtones d'aujourd'hui et les individus qui ont exercé des activités traditionnelles ou qui ont occupé un territoire dans le passé ${ }^{33}$. Or, il faut savoir que la jurisprudence concernant les droits ancestraux ou issus de traité a évolué. Comme nous allons le voir ci-dessous, la Cour suprême du Canada a cherché à trouver un point d'équilibre entre une attitude laxiste et des critères exagérément restrictifs $^{34}$, qu'il s'agisse du rattachement aux peuples ayant vécu aux

31. R. c. Sioui, op. cit., 1058-1059; voir Franklin S. Gertler et Peter W. Hutchins, «Introduction: The Marriage of History and Law in R. v. Sioui», Native Studies Review, 6,2 (1990): 115130 et «Factum of the Intervenor: National Indian Brotherhood (Assembly of First Nations)», Native Studies Review, 6,2 (1990): 132-150.

32. R. c. Sioui, op. cit., 1070.

33. Voir A. Beaulieu, "Les pièges...", op. cit.

34. Voir notamment R. c. Van der Peet, [1996] 2 R.C.S. 507. 
$\mathrm{XVII}^{\mathrm{e}}$ et XVIII ${ }^{\mathrm{e}}$ siècles, de la continuité dans l'exercice d'activités ancestrales ou encore de l'occupation d'un territoire. Cette conception peut bien entendu être critiquée, mais elle constitue une politique judiciaire sur une question que les textes constitutionnels et la jurisprudence antérieure à 1982 passent sous silence.

En ce qui concerne la continuité entre les Autochtones d'aujourd'hui et leurs ancêtres, la Cour suprême a déclaré qu’un lien généalogique peut être déduit ou présumé. Ainsi, dans l'arrêt Simon c. La Reine ${ }^{35}$, l'accusé, un Indien Micmac, est accusé d'avoir eu en sa possession une carabine et des cartouches non loin de la réserve où il habite. En défense, il invoque le droit de chasser protégé par un traité signé en 1752 entre le gouverneur de la Nouvelle-Écosse et les chefs Micmacs habitant la Côte est de cette province. Là encore, la Cour suprême du Canada lui donne raison. Or, selon le gouvernement provincial, l'accusé n’a pas prouvé qu'il est un descendant direct des bénéficiaires du traité. Toutefois, il est membre d'une bande indienne qui porte le même nom que la tribu signataire et qui vit dans la même région. Le juge en chef Dickson règle la question de la manière suivante ${ }^{36}$ :

Il est vrai que cet élément de preuve ne constitue pas une preuve concluante que l'appelant est un descendant direct des Indiens micmacs visés par le Traité de 1752. Toutefois, il doit être suffisant, sinon aucun Indien micmac ne serait en mesure d'établir sa descendance. Les Micmacs ne tenaient aucun registre. Les traditions micmaques sont en grande partie de nature orale. L'imposition d'un fardeau impossible enlèverait effectivement toute valeur au droit de chasse qu'un Indien micmac Subenacadie aurait par ailleurs le droit d'invoquer en se fondant sur ce traité.

Il convient sans doute de préciser que la Loi constitutionnelle de 1982 reconnaît et confirme les droits ancestraux ou issus de traités des peuples autochtones du Canada, expression qui s'entend notamment des Indiens, des Inuit et des Métis ${ }^{37}$. Seuls les premiers sont régis par la Loi sur les Indiens, qui a créé au $\mathrm{xx}^{\mathrm{e}}$ siècle un système de registres. De 1851 à 1985, diverses lois ont également imposé aux Indiens des règles de filiation patrilinéaire (sauf pour les enfants naturels) en privant de leur statut les femmes autochtones ayant épousé un non-Autochtone; si certaines communautés ont réclamé ce changement, d’autres s’y sont

\footnotetext{
35. Simon c. La Reine, [1985] 2 R.C.S. 387.

36. Ibid., 407-408.

37. Article 35 (1) et (2) de la Loi constitutionnelle de 1982, op. cit.
} 
d'ailleurs fortement opposé ${ }^{38}$. Or, ces règles ne peuvent être utilisées pour déterminer l'appartenance aux peuples autochtones reconnus par la Constitution. Dans ces conditions, d'un point de vue strictement juridique, il n'est pas nécessaire de décider si les Innus ont été décimés au $\mathrm{XVII}^{\mathrm{e}}$ siècle par les épidémies et s'ils se sont reconstitués par la suite grâce à des mariages mixtes ou à des adoptions. Ils étaient parfaitement libres d'agir ainsi à l'époque et ils n'ont pas pour autant perdu leur statut de peuple autochtone, comme semble le supposer Russel Bouchard dans la conclusion de son ouvrage de $1995^{39}$ :

[...] en 1845 , les recenseurs de l'état colonial avaient déjà établi que la population aborigène du Bas-Canada n'était désormais qu’une «nation» de Métis et qu'il n'était pratiquement plus possible d'y trouver «un seul sauvage pur sang». Le constat est brutal, voire même cruel pour ces gens qui se disent appartenir aux «Premières Nations » et qui essaient de redéfinir une place au soleil en tentant d'isoler en éprouvette les derniers liens génétiques les rattachant à la sueur de cette terre d'Amérique et en imposant à la communauté toute entière une sorte de politique d'apartheid au profit d'une infime minorité.

Que l'on se rassure: à notre connaissance, aucun tribunal n'a encore exigé des Autochtones la preuve d'un lien génétique s'étendant sur plus de quatre siècles... Ainsi, à propos des Métis, la Cour suprême du Canada requiert la preuve de «liens ancestraux» avec une communauté historique métisse, mais aucun «liens de sang minimums»; l'appartenance à cette collectivité peut être établie par la naissance, par l'adoption " ou autrement ${ }^{40}$ ». Par ailleurs, le fait que celle-ci soit devenue «invisible» du milieu du xix ${ }^{\mathrm{e}}$ siècle au début des années 1970 ne signifie pas qu'elle a disparu ou qu'elle a cessé d'exister. En effet, les historiens agissant comme experts ont expliqué ce phénomène par la réticence des Métis à s'identifier comme tel à cette époque ${ }^{41}$.

Outre cette question d'appartenance, les droits ancestraux présupposent une forme de continuité dans le temps ${ }^{42}$. En effet, l'activité

38. Alain Beaulieu, Les Autochtones du Québec (Québec/Montréal, Musée de la Civilisation/ Fides, 1997), 113; M. Morin, L'usurpation..., op. cit., 220-222.

39. Russel Bouchard, Le dernier des Montagnais. Vie et mort de la nation Ilnu (Chicoutimi-Nord, Russel Bouchard éditeur, 1995), 201.

40. R. c. Powley, 2003 CSC 43, par. 32.

41. Ibid., par. 24-28.

42. Les directives résumées dans ce paragraphe proviennent de $R$. c. Van der Peet, op. cit., par. 64-65. 
exercée de nos jours doit pouvoir être rattachée à une pratique, à une coutume ou à une tradition existant antérieurement à l'arrivée des Européens et faisant partie de la culture distinctive du peuple autochtone concerné. Bien qu'elle ne puisse pas avoir pris naissance après le premier contact, elle peut avoir évolué avec le temps. En effet, la Cour suprême a résolument rejeté la théorie des droits figés, pour éviter par exemple qu'un droit de chasse doive être exercé aujourd'hui de la même manière qu'au $\mathrm{XvIII}^{\mathrm{e}}$ siècle. Par ailleurs, certaines activités ancestrales, comme la pêche, doivent continuer de s'exercer là où elles l'ont été dans le passét3.

Il n'est cependant pas nécessaire de faire la preuve d'une continuité parfaite; à cet égard, la Cour suprême a invité les juges de première instance à faire preuve de souplesse. Une coutume, pratique ou tradition peut avoir été interrompue et reprise ultérieurement. Cela ne constitue pas un obstacle insurmontable à la reconnaissance d'un droit ancestral, à moins que le délai ne soit si prolongé qu'il soit concevable de parler d'un abandon. Ainsi, un grand nombre d'Algonquins ont quitté le bassin de la rivière des Outaouais à la suite de la guerre de 1632 contre les Iroquois; ils l'ont occupé de nouveau à partir de 1666. La Cour suprême leur a néanmoins reconnu un droit ancestral de pêcher pour se nourrir dans cette région, car il était exercé avant 1603, la date du premier contact avec les Européens ${ }^{44}$. En revanche, certains Micmacs se sont établis sur l'île de Terre-Neuve vers 1550, mais leur premier contact avec les Européens date du début du $\mathrm{xvI}^{\mathrm{e}}$ siècle. C’est pourquoi une décision de première instance rejette leur revendication d'un droit ancestral à cet endroit ${ }^{45}$.

Des principes différents s'appliquent au titre aborigène (appelé également titre indien ou titre ancestral $)^{46}$. Celui-ci exige la preuve de l'occupation exclusive d'un territoire au moment de l'affirmation de la souveraineté de la couronne. Dans ce contexte, le groupe qui revendique le titre peut se fonder sur la relation de continuité entre l'occupation actuelle et la situation antérieure à la souveraineté. Là encore, la souplesse est de mise. Selon le juge en chef Lamer, l'occupation et l'utilisation du territoire peuvent avoir été interrompues «à cause peut-être de

43. R. c. Adams, [1996] 3 R.C.S. 101.

44. R. c. Côté, [1996] 3 R.C.S. 139, par. 64 et 69-70.

45. Newfoundland (Minister of Government Services and Lands) c. Drew, op. cit.

46. Voir Delgamuukw c. Colombie-Britannique, [1997] 3 R.C.S. 1010, par. 152-159. 
la réticence des colonisateurs européens à reconnaître le titre aborigène $^{47}$ ». Elles peuvent également avoir évolué. Il suffit donc qu'il y ait eu jusqu’à nos jours "maintien substantiel du lien» entre le peuple et le territoire $^{48}$. Toutefois, il s'agit là d'un mode de preuve mis à la disposition des Autochtones plutôt que d'une exigence à respecter ${ }^{49}$. En effet, depuis l'affirmation de la souveraineté, une partie du territoire visé par le titre aborigène peut avoir cessé d'être occupée par les Autochtones. Mais si aucun acte officiel valide du gouvernement n'est venu l'éteindre, logiquement, il subsiste encore.

Le caractère exclusif de la possession n'a pas non plus à être absolu. En effet, il peut être établi en dépit d'intrusions qui ont été réprouvées par les Autochtones ou de la présence d'étrangers qui ont été autorisés à séjourner sur le territoire ou à s'y établir. Par ailleurs, le nomadisme est peut-être inconciliable avec une telle possession. La Cour suprême s'est contenté de formuler cette dernière question sans y apporter de réponse $^{50}$; récemment, les cours d'appel du Nouveau-Brunswick et de la Nouvelle-Écosse ont cependant écarté cette thèse ${ }^{51}$. Dans ces conditions, un peuple autochtone qui quitte son territoire ancestral et qui s'établit ailleurs pourrait se voir reconnaître un nouveau titre aborigène. Là encore, certains juges de la Cour suprême ont fait allusion à ce problème, mais sans prendre parti ${ }^{52}$. Il est donc inexact ou prématuré d'affirmer qu'une migration est incompatible avec l'existence d'un titre territorial, comme le fait Alain Beaulieu ${ }^{53}$.

Pourtant, Nelson-Martin Dawson affirme que la disparition de peuples entiers ou leur migration prouvent l'inexistence de leurs droits: « $\mathrm{Si}$, au contraire, ces Attikamègues et ces Têtes-de-Boule avaient toujours constitué deux communautés autochtones distinctes, dont les

47. Ibid., par. 153 .

48. Id.

49. R. c. Bernard, [2003] N.B.J. Nº 320 (C.A.N.-B.) (QL Systems), requête en autorisation de pourvoi à la Cour suprême du Canada (no 3005), par. 57-59 et 131-135 (j. Daigle) et par. 445 (j. Robertson); R. c. Marshall, [2003] N.S.J. N. 361 (C.A.N.-É.) (QL Systems), requête en autorisation de pourvoi à la Cour suprême du Canada (n 30063), par. 157-181 (j. Cromwell, avec l'accord des juges Oland et Saunders (par. 257).

50. Delgamuukw c. Colombie-Britannique, op. cit., par. 139.

51. R. c. Bernard, op. cit., par. 84-90(j. Daigle) et par. 445 (j. Robertson); R. c. Marshall, op. cit., par. 187-194 (j. Cromwell, avec l'accord des juges Oland et Saunders (par. 257).

52. Delgamuukw c. Colombie-Britannique, supra, note 45, par. 197-198 (opinion minoritaire du juge La Forest).

53. A. Beaulieu, «Les pièges...», op. cit., 551. 
seconds s'étaient substitué aux premiers au cours des siècles, la discontinuité d'occupation serait prouvée et infirmerait, pour les occupants autochtones actuels, cette même notion de titre indien de Commun [sic] $L a w^{54}$." Il est difficile d'échapper à la conclusion que ces analyses reposent sur une méconnaissance des exigences posées par la Cour suprême depuis $1996^{55}$. Dans le cas de l'ouvrage de M. Dawson, cela s'explique peut-être par le fait qu'il s'agit d'un rapport réalisé pour le compte d'Hydro-Québec, vraisemblablement avant que les arrêts en question n'aient été rendus ${ }^{56}$.

En effet, en Colombie-Britannique, la Cour suprême du Canada a conclu que l'affirmation de la souveraineté britannique remonte à 1846, l'année du traité de l'Oregon conclu entre les États-Unis et la GrandeBretagne $^{57}$. Pour la péninsule de la Nouvelle-Écosse, devant la Cour d'appel de cette province, les parties ont accepté que la souveraineté date de 1713, date du traité d'Utrecht, tandis qu'au Cap Breton, l'année du traité de Paris (1763) a été retenue ${ }^{58}$. Au Nouveau-Brunswick, c'est plutôt la date de la conquête (1759) qui a été acceptée ${ }^{59}$. Dans le cas du Québec, l'affirmation de la souveraineté semble également devoir être fixée à $1763^{60}$. En effet, cette année-là, la France cède le Canada à la GrandeBretagne par le traité de Paris; puis, la Proclamation royale reconnaît officiellement le titre aborigène, ce qui n'avait jamais été le cas sous le Régime français ${ }^{61}$. Si tel est le cas, il importe peu que, vers la fin du $\mathrm{xvII}^{\mathrm{e}}$

54. Nelson-Martin Dawson, Des Attikamègues aux Têtes-de-Boule. Mutation ethnique dans le Haut Mauricien sous le Régime français (Sillery, Septentrion, 2003), 10.

55. Voir notamment R. c. Van der Peet, op. cit.; Delgamuukw c. Colombie-Britannique, op. cit.

56. Claude Gélinas, «Des Attikamègues aux Têtes de Boule. Mutation ethnique dans le Haut Mauricien sous le Régime français, Nelson-Martin Dawson, Septentrion, Sillery, 2003. 171 pages » (compte rendu), Recherches amérindiennes au Québec, 23,2 (2003): 139; Alain Beaulieu, "Les pièges...", op. cit., 543 .

57. Delgamuukw c. Colombie-Britannique, op. cit., par. 145.

58. R. c. Marshall, op. cit., par. 73 (j. Cromwell, avec l'accord des juges Oland et Saunders (par. 257).

59. R. c. Bernard, op. cit., par. 61 (j. Daigle) et par. 445 (j. Robertson).

60. En ce qui concerne le titre aborigène, la Cour suprême ne s'est pas prononcée sur cette question, mais elle n'a pas commenté le raisonnement des juges d'instance inférieure faisant remonter la souveraineté à $1763:$ R. c. Adams, op. cit., par. 18 et 46; R. c. Côté, op. cit., par. 15-21, 49 et 60 .

61. Voir notamment Andrée Lajoie, Jean-Maurice Brisson, Sylvio Normand et Alain Bissonnette, Le statut juridique des peuples autochtones au Québec et le pluralisme (Cowansville, Éditions Yvon Blais, 1996); M. Morin, L'Usurpation..., op. cit., chap. 3 ; Michel Morin, " "Manger avec la même micoine dans la même gamelle" : à propos des traités conclus avec les Amérindiens au Québec, 1665-1760", Revue générale de droit, 33 (2003): 93-129. 
siècle, les Têtes-de-Boule aient quitté leurs territoires situés à l'ouest pour venir s'établir en Haute-Mauricie; en effet, cela ne saurait constituer un obstacle à la reconnaissance d'un titre aborigène à compter de $1763^{62}$.

Par ailleurs, la Cour suprême utilisera dorénavant un troisième point de repère temporel. En effet, outre le contact avec les Européens pour les droits ancestraux et l'affirmation de la souveraineté pour le titre aborigène, dans le cas des Métis, elle se fonde sur «l'antériorité à la mainmise effective des Européens sur le territoire», en tenant compte du moment où ceux-ci «ont effectivement établi leur domination politique et juridique dans une région donnée ${ }^{63} \%$. Ces variations dans le temps ne font l'objet d'aucune justification convaincante ${ }^{64}$ et conduisent à des résultats absurdes. Ainsi, à Terre-Neuve, les Micmacs auraient pu acquérir un titre aborigène avant l'affirmation de la souveraineté ; une communauté métisse aurait pu exercer des droits ancestraux avant la mainmise des Européens sur le territoire; mais les Micmacs ne peuvent en détenir en raison de leur installation après le contact ${ }^{65}$.

Tout cela pour dire que la preuve de l'exercice d'une activité ou de l'occupation exclusive doit remplir des conditions fixées par la jurisprudence contemporaine. En ce domaine, l'Histoire ne peut fournir de réponse, même si elle constitue le matériau qu'utilisent les juges. Les historiens sont certes libres d'être en désaccord avec la démarche que ceux-ci adoptent, mais ils devraient au moins la critiquer à bon escient.

\section{LA MAXIMISATION DES ÉLÉMENTS HISTORIQUES}

L'interprétation des documents anciens nécessite le plus souvent une mise en contexte que seuls les historiens sont à même de fournir. Mais cette contribution peut parfois avoir une influence démesurée sur l'esprit des juges, qui confondent parfois la portée d'un texte normatif et les préoccupations concrètes de ses auteurs. Réciproquement, la qualification juridique de relations historiques complexes est inévitablement colorée par les préoccupations contemporaines des auteurs prenant position sur ces questions.

62. En d'autres termes, en admettant que la démonstration de M. Dawson soit réussie sur le plan historique, elle rate totalement sa cible sur le plan juridique (N.-M. Dawson, Des Attikamègues..., op. cit.).

63. R. c. Powley, op. cit., par. 37.

64. Voir Brian Slattery, "Making Sense of Aboriginal and Treaty Rights», Revue du Barreau Canadien, 79 (2000): 196.

65. Newfoundland (Minister of Government Services and Lands) c. Drew, op. cit. 


\section{La perception de l'époque et la Proclamation royale}

Au-delà de la reconstitution des événements jugés pertinents, la méthode historique privilégie l'analyse des relations sociales ainsi que des motivations et des valeurs qui animent les acteurs de la période étudiée. Dans cette perspective, il est possible de se demander quelle était la conception des administrateurs coloniaux et des peuples autochtones. Mais dans le domaine juridique, il existe un risque de donner une portée trop absolue à la vision de ces individus. Il en va ainsi de l'interprétation de la Proclamation royale de 1763 . Celle-ci protège les «terres qui, n’ayant pas été cédées au roi ni achetées par lui, sont réservées » aux Autochtones à titre de territoires de chasse. En raison de la rédaction tortueuse du texte, il est cependant difficile de déterminer s'il existe de telles terres à l'intérieur du Québec et de la Nouvelle-Écosse de l'époque, par opposition au territoire réservé aux Autochtones à l'ouest et au nord de la ligne de partage des eaux, qu'on appelle couramment le territoire Indien.

Des historiens ont donc conclu que la partie de la Proclamation concernant les territoires de chasse ne s'applique pas à la NouvelleÉcosse $^{66}$ et au Québec ${ }^{67}$, en se fondant sur les documents préparatoires et sur la pratique contemporaine des autorités. Plusieurs juristes québécois ont toutefois contesté cette interprétation réductrice ${ }^{68}$. On constate alors avec étonnement que MM. Delâge et Sawaya reprennent la première de ces thèses sans même mentionner l'autre ${ }^{69}$. Il est encore plus étonnant de lire, à propos d'une éventuelle cession de territoire, que "l'hypothèse d'un refus des autochtones n'est pas reconnue» par la Proclamation royale, sous le couvert d'une «interprétation historique [...] et non pas juridique $^{70} »$. Pourtant, ce document exige que les Autochtones soient «enclins» à «céder» leurs terres avant que le gouverneur puisse convoquer une assemblée publique pour en discuter. Implicitement, il leur reconnaît donc la possibilité de refuser la cession ${ }^{71}$.

66. Jack Stagg, Anglo-American Relations in North America to 1763 and An Analysis of the Royal Proclamation of 7 October 1763 (Ottawa, Research Branch, Indian and Northern Affairs, 1981), 270277.

67. Jacqueline Beaulieu, Christiane Cantin et Maurice Ratgelle, «La Proclamation royale de 1763 : le droit refait l'histoire», Revue du Barreau, 51 (1989): 317.

68. Paul Dionne, «Les postulats de la Commission Dionne et le titre aborigène au Québec: vingt ans après », R. du B., 51 (1991): 127; Richard Boivin, "Pour en finir avec la Proclamation royale : la décision Côté», Revue générale de droit, 25 (1994): 131; M. Morin, L'usurpation..., op. cit., 136-145.

69. D. Delâge et J.-P. Sawaya, op. cit., 104.

70. Ibid., 14.

71. Ibid., 101. Le texte de la Proclamation est reproduit aux pages 100.1 à 100.4 
Pour en revenir au débat concernant la Proclamation royale, les cours d'appel du Québec et de la Nouvelle-Écosse ont accepté l'opinion des historiens, en interprétant ce texte en fonction des perceptions contemporaines des auteurs du document ${ }^{72}$, tandis que la Cour suprême du Canada a refusé de prendre position ${ }^{73}$. La Cour d'appel du NouveauBrunswick a privilégié une autre approche, qui nous semble personnellement plus appropriée. En effet, le juge Robertson souligne que l'interprétation d'un document juridique est une question de droit. Celle-ci doit être tranchée par le tribunal plutôt que par les historiens agissant à titre d'expert, même si leur contribution est inestimable pour replacer un écrit dans son contexte ${ }^{74}$. Le juge Robertson rejette alors l'opinion du professeur Patterson, selon qui la Proclamation protège uniquement les territoires autochtones qui ont déjà été réservés par un acte officiel provenant des représentants de la couronne. Car si tel est le cas, ce texte devient superflu. En définitive, il conclut que, en l'absence de cession ou de vente, les territoires de chasse dont les Autochtones de Nouvelle-Écosse étaient en possession en 1763 leur ont été réservés ${ }^{75}$.

\section{L'influence des positions contemporaines}

La tentation de projeter nos valeurs et nos préoccupations dans le passé est inhérente à la recherche historique. Bien plus que les historiens, les juristes sont passés maîtres dans l'art de détacher un extrait de son contexte pour se l'approprier et lui donner une signification nouvelle. La pratique est courante dans la plaidoirie et la rédaction de motifs. Mais les historiens ne résistent pas toujours à la tentation de conclure que certaines revendications actuelles n'ont pas de fondement historique. Certes, ils ont le droit de prendre position sur des questions controversées. Mais leurs affirmations peuvent parfois être tendancieuses. Ainsi, certains se sont demandé si Louis XIV pouvait même concevoir que des peuples autochtones aient un droit de propriété sur leurs territoires, parce qu'à l'époque, l'on déniait tout droit de propriété au paysan français ${ }^{76}$. Quelle que soit la conception du roi de France sur la question, l’argument invoqué pour suggérer au lecteur la réponse n’a pas

72. Côté c. La Reine, [1993] R.J.Q. 1350, 1361-1364 (C.A.); R. c. Marshall, op. cit., par. 196-226 (j. Cromwell, avec l'accord des juges Oland et Saunders (par. 257).

73. R. c. Côté, op. cit., par. 41.

74. R. c. Bernard, op. cit., par. 391 (j. Robertson), avec l'accord du juge Daigle (par. 186-189).

75. Ibid., par. 412-428. Le juge Robertson précise que la possession doit être prouvée de la même manière que l'occupation pour les fins du titre aborigène (par. 441-444).

76. N.-M. Dawson et E. Tremblay, "La preuve historique...», op. cit., 382. 
vraiment de fondement historique. Dans le cadre du régime seigneurial, largement dominant dans la France de l'époque, le seigneur avait la propriété éminente de la terre tandis que le censitaire, souvent un paysan, en avait la propriété utile. À toutes fins utiles, le censitaire pouvait la vendre, l'échanger ou la transmettre comme s'il en était l'unique propriétaire. Pour la lui retirer, le seigneur devait obtenir un jugement, ce qui était devenu fort rare au $\mathrm{xvII}^{\mathrm{e}}$ siècle ${ }^{77}$.

En ce qui concerne les droits des peuples autochtones, dans certains cas, il est difficile d'analyser de manière objective et détachée les affirmations contradictoires qu'on retrouve dans la documentation historique. La question de l'indépendance ou de l'assujettissement à la couronne des membres de la Confédération haudenosaunee (anciennement appelée iroquoise) constitue un bon exemple de ce problème. La position des Britanniques varie selon les personnages et les époques. La thèse de la soumission repose sur des documents unilatéraux ou sur des écrits qui attribuent aux Haudenosaunee des propos qu'ils n'ont pas toujours tenus ${ }^{78}$. Dans ces conditions, un historien peut fort bien conclure qu'il n'existe aucune volonté commune lors des négociations pertinentes, encore qu'un négociateur comme William Johnson défende énergiquement devant ses supérieurs la thèse de l'indépendance de la Confédération.

Mais la relation établie au fil des siècles, qualifiée de "chaîne du covenant», a indubitablement existé. S'il faut lui donner un contenu normatif de nos jours, il est essentiel de trouver un terrain mitoyen plutôt que de choisir entre des positions incompatibles. Par exemple, il pourrait s'agir d'une autonomie interne dans le cadre de l'État canadien ${ }^{79}$. La difficulté est d'ailleurs la même lorsque vient le temps de préciser la signification contemporaine des engagements contenus dans les traités. Quant à la fiction que représente la souveraineté de la couronne sur les territoires autochtones, au $\mathrm{XVII}^{\mathrm{e}}$ et au $\mathrm{XvIII}^{\mathrm{e}}$ siècles, elle a selon nous une portée internationale qui n'exclut d'aucune manière le maintien initial

77. Jean Bart, Histoire du droit privé, de la chute de l'Empire romain au XIX ${ }^{e}$ siècle (Paris, Montchrestien, 1998), 257-259; Louise Dechêne, Habitants et marchands de Montréal au XVII siècle, essai (Montréal, Boréal, 1988), 255-256; A. Lajoie et al., op. cit., 127-130 et 254-258; Olivier Martin, Histoire de la Coutume de la prévôté et vicomté de Paris (Paris, Éditions Cujas, 1972), 1: 448. 78. Voir Mark D. Walters, «Brightening the Covenant Chain: Aboriginal Treaty Meanings in Law and History after Marshall», Dalhousie Law Journal, 24 (2001: 75-138, 97-107.

79. Ibid., 125-138. 
des ordres juridiques autochtones ${ }^{80}$. Mais il n'est guère possible de discuter de ces questions en faisant totalement abstraction de ses opinions sur les revendications actuelles des peuples autochtones.

\section{CONCLUSION}

La lecture que les tribunaux font du passé est destinée à trouver des solutions aux problèmes actuels, en écartant de l'analyse les éléments qui sont incompatibles avec les principes fondamentaux du système juridique canadien. Ainsi, au $\mathrm{XvII}^{\mathrm{e}}$ et au $\mathrm{XvIII}^{\mathrm{e}}$ siècles, la violation d'un traité est sanctionnée par le recours aux armes ou par l'interruption des échanges entre les partenaires. Seul un processus de négociation permet aux Autochtones de présenter leurs doléances et d'obtenir satisfaction. À cette époque, ils peuvent pratiquer la chasse et la pêche librement. Puis, lorsque le rapport de force tourne à l'avantage de l'État, celui-ci impose unilatéralement son autorité, notamment en réglementant ces activités. Depuis 1982, les droits ancestraux ou issus de traité sont protégés par la Constitution. Ils ont préséance sur la législation, dans les limites fixées par les tribunaux. Le contexte a donc changé du tout au tout. C'est pourquoi les décisions rendues par les juges ont peu à voir avec les préoccupations contemporaines des parties au traité. Mais ce décalage est inhérent à l'analyse juridique : par la force des choses, une convention ordinaire ou une disposition législative peuvent donner lieu à des difficultés auxquelles personne n'avait songé. Les tribunaux sont alors laissés à eux-mêmes et doivent façonner une solution, en s'inspirant des attentes des cocontractants ou du législateur. Ils ne procèdent pas autrement en matière de droits autochtones, même s'il existe un hiatus beaucoup plus considérable entre le passé et le présent. C'est sans doute ce qui explique l'incompréhension voire l'agressivité que manifestent parfois certains historiens à l'égard de leurs décisions.

80. Ibid., 87-89 et 106-107; M. Morin, L'usurpation..., op. cit., 106-119, 154-155 et 194-200; William J. Eccles, "Sovereignty-Association, 1500-1783», Canadian Historical Review, 45 (1984): 475-495 ; P. C. McHugh, "The Common-Law Status of Colonies and Aboriginal "Rights" : How Lawyers and Historians Treat the Past», Sask.L.R., 61 (1998): 393 ; Mark Walters, "The "Golden Thread" of Continuity: Aboriginal Customs at Common Law and Under the Constitution Act, 1982 », Revue de droit McGill, 44 (1999): 711 ; voir André Émond, "L’inadéquation entre le droit et l'histoire", Revue de droit de l'Université de Sherbrooke, 33 (2002-03): 317-376. 